\title{
Scattering problem for Klein-Gordon equation with cubic convolution nonlinearity *
}

\author{
Ruying Xue ${ }^{\dagger}$ \\ Department of Mathematics, Zhejiang University, \\ Hangzhou 310027, Zhejiang, P. R. China
}

\begin{abstract}
The scattering problem for the Klein-Gordon equation with cubic convolution nonlinearity is considered. Based on the Strichartz estimates for the inhomogeneous KleinGordon equation, we prove the existence of the scattering operator, which improves the known results in some sense.
\end{abstract}

Keywords: Asymptotic of solution; Klein-Gordon equation; scattering operator

Subject class: 35P25, 81Q05, 35B05

\section{Introduction}

This paper is concerned with the scattering problem for the nonlinear Klein-Gordon equation of the form

$$
\left\{\begin{array}{l}
\partial_{t}^{2} u-\Delta u+u=F_{\gamma}(u) \quad(t, x) \in R \times R^{n} \\
\left.u\right|_{t=0}=f(x),\left.\partial_{t} u\right|_{t=0}=g(x)
\end{array}\right.
$$

where $\mathrm{u}$ is a real-valued or a complex-valued unknown function of $(t, x) \in R \times R^{n}$. The nonlinearity is a cubic convolution term $F_{\gamma}(u)=-\left(V_{\gamma}(x) *|u|^{2}\right) u$ with $\left|V_{\gamma}(x)\right| \leq C|x|^{-\gamma}$. Here, $0<\gamma<n$ and $*$ denotes the convolution in the space variables. The term $F_{\gamma}(u)$ is an approximative expression of the nonlocal interaction of specific elementary particles. The equation (1.1) was studied by Menzala and Strauss in [1].

In order to define the scattering operator for (1.1), we first give some Banach spaces. The usual Lebesgue space is given by $L^{p}=\left\{\phi \in S^{\prime}:\|\phi\|_{L^{p}}<+\infty\right\}$, where the norm $\|\phi\|_{L^{p}}=\left\{\int_{R^{n}}|\phi(x)| d x\right\}^{1 / p}$ if $1 \leq p<+\infty$ and $\|\phi\|_{L^{\infty}}=\sup _{x \in R^{n}}|\phi(x)|$ if $p=+\infty$. The weighted Sobolev space $H_{p}^{\beta, k}$ is defined by

$$
H_{p}^{\beta, k}=\left\{\phi \in S^{\prime}:\|\phi\|_{H_{p}^{\beta, k}}=\left\|\langle x\rangle^{k}\langle i \nabla\rangle^{\beta} \phi\right\|_{L^{p}}<+\infty\right\}
$$

with $\langle x\rangle=\sqrt{1+x^{2}}$ and $\langle i \nabla\rangle=\sqrt{1-\triangle}$. We also write $H^{\beta, k}=H_{2}^{\beta, k}$ and $H^{\beta}=H_{2}^{\beta, 0}$ if it does not cause a confusion. A Hilbert space $X^{\beta, k}$ is denoted by $H^{\beta, k} \bigoplus H^{\beta-1, k}$. Let $X_{\rho}^{\beta, k}$ be a ball of a radius $\rho>0$ with a center in the origin in the space $X^{\beta, k}$. The scattering operator of (1.1) is defined as the mapping $S: X_{\rho}^{\beta, k} \ni\left(f_{-}, g_{-}\right) \rightarrow\left(f_{+}, g_{+}\right) \in X^{\beta, 0}$ if the following condition holds:

${ }^{*}$ Supported by NSFC (10931007) and Zhejiang Provincial NSF of China (Y6090158)

${ }^{\dagger}$ E-mail address: ryxue@zju.edu.cn 
For $\left(f_{-}, g_{-}\right) \in X_{\rho}^{\beta, k}$, there uniquely exists a time-global solution $u \in C\left(R ; H^{\beta}\right)$ of (1.1), and data $\left(f_{+}, g_{+}\right) \in X^{\beta, 0}$ such that $u(t)$ approaches $u_{ \pm}(t)$ in $H^{\beta}$ as $t \rightarrow \pm \infty$, where $u_{ \pm}(t)$ are solutions of linear Klein-Gordon equations whose initial data are $\left(f_{ \pm}, g_{ \pm}\right)$, respectively.

We say that $\left(S, X^{\beta, k}\right)$ is well-defined if we can define the scattering operator $S$ : $X_{\rho}^{\beta, k} \rightarrow X^{\beta, 0}$ for some $\rho>0$. In [2], Mochizuki prove that if $n \geq 3, \beta \geq 1, \gamma<n$ and $2 \leq \gamma \leq 2 \beta+2$, then $\left(S, X^{\beta, 0}\right)$ is well-defined. Hidano [3] see that if $n \geq 2, \beta \geq 1$, $4 / 3<\gamma<2$ and $k>1 / 3$, then $\left(S, X^{\beta, k}\right)$ is well-defined. By using the Strichartz estimate for pre-admissible pair and the complex interpolation method for the weighted Sobolev space, Hidano [4] shows that $\left(S, X^{\beta, k}\right)$ is well-defined if $n \geq 2, \beta \geq 1,4 / 3<\gamma<2$ and $k>(2-\gamma) / 2$. Our aim of this article is to show that $\left(S, X^{\beta, 1}\right)$ is well-defined if $n \geq 2$,

$$
1<\gamma<\min \left\{\frac{2(n+1)}{n+2}, \frac{3 n-2}{n+2}\right\}, \frac{(n+2)(\gamma+1)}{4 n}+\frac{1}{2}<\beta<\frac{(n+2)(\gamma+1)}{2 n} .
$$

More precisely, we prove the following theorem.

Theorem 1.1 Let the function $V_{\gamma}(x)$ satisfy

$$
\left|V_{\gamma}(x)\right| \leq C|x|^{-\gamma},\left|\nabla V_{\gamma}(x)\right| \leq C|x|^{-(1+\gamma)} .
$$

Assume that $n \geq 2, \gamma$ and $\beta$ satisfy (1.2). Then there exists a positive number $\delta_{0}>0$ satisfying the following properties:

(1). For $(f, g) \in X^{\beta, 1}$ with $\|(f, g)\|_{X^{\beta, 1}} \leq \delta_{0}$, there uniquely exist finial states $\left(f_{ \pm}, g_{ \pm}\right) \in$ $X^{\beta, 0}$ and a solution $u(t) \in C\left(R ; H^{\beta}\right)$ of (1.1) such that $u(t)$ approaches $u_{ \pm}(t)$ in $X^{\beta, 0}$ as $t \rightarrow \pm \infty$, where $u_{ \pm}(t)$ are solutions of the linear Klein-Gordon equation with initial data $\left(f_{ \pm}, g_{ \pm}\right)$, respectively. Moreover, as $\pm t$ large enough we have

$$
\left\|\left(u(t), \partial_{t} u(t)\right)-\left(u_{ \pm}(t), \partial_{t} u_{ \pm}(t)\right)\right\|_{X^{\beta, 0}} \leq C\langle t\rangle^{-\delta}
$$

with $\delta=\frac{2 n \beta}{n+2}-2>0$.

(2). For $\left(f_{-}, g_{-}\right) \in X^{\beta, 1}$ with $\left\|\left(f_{-}, g_{-}\right)\right\|_{X^{\beta, 1}} \leq \delta_{0}$, there uniquely exists a finial state $\left(f_{+}, g_{+}\right) \in X^{\beta, 0}$ and a solution $u(t) \in C\left(R ; H^{\beta}\right)$ of (1.1) such that $u(t)$ approaches $u_{ \pm}(t)$ in $X^{\beta, 0}$ as $t \rightarrow \pm \infty$, where $u_{ \pm}(t)$ are solutions of the linear Klein-Gordon equation with initial data $\left(f_{ \pm}, g_{ \pm}\right)$, respectively. Moreover, as $\pm t$ large enough we have

$$
\left\|\left(u(t), \partial_{t} u(t)\right)-\left(u_{ \pm}(t), \partial_{t} u_{ \pm}(t)\right)\right\|_{X^{\beta, 0}} \leq C\langle t\rangle^{-\delta}
$$

with $\delta=\frac{2 n \beta}{n+2}-2>0$.

In this article we denote by $J_{\varepsilon}=\langle i \nabla\rangle x+i \varepsilon t \nabla, L_{\varepsilon}=i \partial_{t}-\varepsilon\langle i \nabla\rangle$ and $P=t \nabla+x \partial_{t}$ with $\varepsilon \in\{+,-\}$. For a given Banach space with norm $\|\cdot\|$ and a vector $v=\left(v^{+}, v^{-}\right)$, denote by

$$
\begin{gathered}
\|v\|=\left\|v^{+}\right\|+\left\|v^{-}\right\|,\|P v\|=\left\|P v^{+}\right\|+\left\|P v^{-}\right\|, \\
\|J v\|=\left\|J_{+} v^{+}\right\|+\left\|J_{-} v^{-}\right\|,\|L v\|=\left\|L_{+} v^{+}\right\|+\left\|L_{-} v^{-}\right\| .
\end{gathered}
$$

We also denote by the space-time norm

$$
\|\phi\|_{L_{t}^{r}\left(I, L_{x}^{q}\right)}=\|\| \phi(t)\left\|_{L_{x}^{q}\left(R^{n}\right)}\right\|_{L_{t}^{r}(I)}
$$

where I is a bounded or unbounded time interval, and denote different positive constants by the same letter $\mathrm{C}$.

The rest of the article is organized as follows. In Section 2 we give some preliminary calculations. Then Section 3 is devoted to the proof of Theorem 1.1. 


\section{Preliminaries}

In this section, we prove some lemmas for our main results. Let $w^{\varepsilon}=i \partial_{t}\langle i \nabla\rangle^{-1} u-\varepsilon u$ with $\varepsilon \in\{+,-\}$. Then the Klein-Gordon equation (1.1) can be be rewritten as a system of equations

$$
\left\{\begin{array}{l}
L_{\varepsilon} w^{\varepsilon}=\langle i \nabla\rangle^{-1} F_{\gamma}(u) \\
\left.w^{\varepsilon}\right|_{t=0}=w_{0}^{\varepsilon}
\end{array}\right.
$$

where $L_{\varepsilon}=i \partial_{t}-\varepsilon\langle i \nabla\rangle, w_{0}^{\varepsilon}=i\langle i \nabla\rangle^{-1} g+\varepsilon f$. By the fact that

$$
u=\frac{1}{2}\left(w^{+}-w^{-}\right), \partial_{t} u=-\frac{i}{2}\langle i \nabla\rangle\left(w^{+}+w^{-}\right),
$$

we can rewrite the term $F_{\gamma}(u)$ as

$$
F_{\gamma}(u)=\sum_{\varepsilon_{1}, \varepsilon_{2}, \varepsilon_{3} \in\{+,-\}} C_{\varepsilon_{1} \varepsilon_{2} \varepsilon_{3}}\left(V_{\gamma} * \overline{w^{\varepsilon}} w^{\varepsilon_{2}}\right) w^{\varepsilon_{3}}
$$

with some constants $C_{\varepsilon_{1} \varepsilon_{2} \varepsilon_{3}}$. Denote $U_{\varepsilon}(t) \varphi=e^{-\varepsilon i\langle i \nabla\rangle t} \varphi$ and for given $T \in R$,

$$
\Psi_{\varepsilon}[g]=\int_{T}^{t} U_{\varepsilon}(t-\tau)\langle i \nabla\rangle^{-1} g(\tau) d \tau,
$$

Lemma 2.1 Let $2 \leq q<\frac{2 n}{n-2}, \frac{2}{r}=\frac{n}{2}\left(1-\frac{2}{q}\right)$. Then for any time interval $I$ and for any given $T \in I$ the following estimates are true:

$$
\begin{gathered}
\left\|\Psi_{\varepsilon}[g]\right\|_{L_{t}^{r}\left(I, L^{q}\right)} \leq\|g\|_{L_{t}^{r \prime}\left(I, H_{q^{\prime}}^{2 \mu-1}\right)}, \\
\left\|\Psi_{\varepsilon}[g]\right\|_{L_{t}^{\infty}\left(I, L^{2}\right)} \leq\|g\|_{L_{t}^{r \prime}\left(I, H_{q \prime}^{\mu-1}\right)},
\end{gathered}
$$

and

$$
\left\|U_{\varepsilon}(t) \varphi\right\|_{L_{t}^{r}\left(I, L^{q}\right)} \leq\|\varphi\|_{H^{\mu}}
$$

where $r^{\prime}=\frac{r}{r-1}, q^{\prime}=\frac{q}{q-1}$ and $\mu=\frac{1}{2}\left(1+\frac{n}{2}\right)\left(1-\frac{2}{q}\right)$.

The proof of Lemma 2.1 is based on the duality argument along with the $L^{p}-L^{q}$ time decay estimates. The similar result be found in [5].

Lemma 2.2 Assume $2 \leq p<\frac{2 n}{n-2}$ for $n \geq 3(2 \leq p<+\infty$ for $n=2)$, denote by $\alpha=\left(1+\frac{n}{2}\right)\left(1-\frac{2}{p}\right)$. The estimate is valid

$$
\|\phi\|_{L^{p}} \leq C\langle t\rangle^{-\frac{n}{2}\left(1-\frac{2}{p}\right)}\left(\|\phi\|_{H^{\alpha}}+\left\|J_{\varepsilon} \phi\right\|_{H^{\alpha-1}}\right)
$$

for all $t \in R$, provided that the right-hand side is finite.

This lemma comes from Lemma 2.1 in [5] and the fact that $\|\phi\|_{L^{p}} \leq C\|\phi\|_{H^{\alpha}}$ when $p \geq 2$.

Lemma 2.3 Assume $\left|V_{\gamma}(x)\right| \leq|x|^{-\gamma}$ with $0<\gamma<n, \varepsilon_{1}, \varepsilon_{2}, \varepsilon_{3} \in\{+,-\}$.

(1). For $1<r<+\infty, 1<p_{1}, p_{2}<+\infty$ and $p_{3}>r$ satisfying $1+\frac{1}{r}=\frac{\gamma}{n}+\frac{1}{p_{1}}+\frac{1}{p_{2}}+\frac{1}{p_{3}}$, we have

$$
\left\|\left(V_{\gamma} * \overline{w^{\varepsilon_{1}}} w^{\varepsilon_{2}}\right) w^{\varepsilon_{3}}\right\|_{L^{r}} \leq\left\|w^{\varepsilon_{1}}\right\|_{L^{p_{1}}}\left\|w^{\varepsilon_{2}}\right\|_{L^{p_{2}}}\left\|w^{\varepsilon_{3}}\right\|_{L^{p_{3}}}
$$


(2). For $\rho>0,1<r<+\infty, 1<p_{j k}<+\infty$ for $j, k \in\{1,2\}$ and $p_{13}, p_{23}>r$ satisfying $1+\frac{1}{r}=\frac{\gamma}{n}+\frac{1}{p_{j 1}}+\frac{1}{p_{j 2}}+\frac{1}{p_{j 3}}$, we have

$$
\begin{aligned}
\left\|\left(V_{\gamma} * \overline{w^{\varepsilon_{1}}} w^{\varepsilon_{2}}\right) w^{\varepsilon_{3}}\right\|_{H_{r}^{\rho}} \leq & \left\|w^{\varepsilon_{1}}\right\|_{H_{p_{11}}^{\rho}}\left\|w^{\varepsilon_{2}}\right\|_{L^{p_{12}}}\left\|w^{\varepsilon_{3}}\right\|_{L^{p_{13}}}+\left\|w^{\varepsilon_{1}}\right\|_{L^{p_{12}}}\left\|w^{\varepsilon_{2}}\right\|_{H_{p_{11}}^{\rho}}\left\|w^{\varepsilon_{3}}\right\|_{L^{p_{13}}} \\
& +\left\|w^{\varepsilon_{1}}\right\|_{L^{p_{21}}}\left\|w^{\varepsilon_{2}}\right\|_{L^{p_{22}}}\left\|w^{\varepsilon_{3}}\right\|_{H_{p_{23}}^{\rho}}
\end{aligned}
$$

Proof. To prove (1), put $\frac{1}{p_{4}}=\frac{1}{r}-\frac{1}{p_{3}}$. By the Hölder inequality and the Hardy-LittlewoodSobolev inequality, we have

$$
\begin{aligned}
\left\|\left(V_{\gamma} * \overline{w^{\varepsilon_{1}}} w^{\varepsilon_{2}}\right) w^{\varepsilon_{3}}\right\|_{L^{r}} & \leq\left\|V_{\gamma} * \overline{w^{\varepsilon_{1}}} w^{\varepsilon_{2}}\right\|_{L^{p_{4}}}\left\|w^{\varepsilon_{3}}\right\|_{L^{p_{3}}} \\
& \leq\left\|w^{\varepsilon_{1}}\right\|_{L^{p_{1}}}\left\|w^{\varepsilon_{2}}\right\|_{L^{p_{2}}}\left\|w^{\varepsilon_{3}}\right\|_{L^{p_{3}}}
\end{aligned}
$$

since $1+\frac{1}{p_{4}}=\frac{\gamma}{n}+\frac{1}{p_{1}}+\frac{1}{p_{2}}$.

To prove (2), we set $\frac{1}{r}=\frac{1}{p_{14}}+\frac{1}{p_{13}}$ and $\frac{1}{r}=\frac{1}{p_{24}}+\frac{1}{p_{23}}$. For $\rho>0$, the generalized Hölder inequality in [6] implies

$$
\left\|\left(V_{\gamma} * \overline{w^{\varepsilon_{1}}} w^{\varepsilon_{2}}\right) w^{\varepsilon_{3}}\right\|_{H_{r}^{\rho}} \leq\left\|V_{\gamma} * \overline{w^{\varepsilon_{1}}} w^{\varepsilon_{2}}\right\|_{H_{p_{14}}^{\rho}}\left\|w^{\varepsilon_{3}}\right\|_{L^{p_{13}}}+\left\|V * \overline{w^{\varepsilon_{1}}} w^{\varepsilon_{2}}\right\|_{L^{p_{24}}}\left\|w^{\varepsilon_{3}}\right\|_{H_{p_{23}}^{\rho}}
$$

By the generalized Hölder inequality and the Hardy-Littlewood-Sobolev inequality, we have

$$
\begin{aligned}
\left\|V_{\gamma} * \overline{w^{\varepsilon}} w^{\varepsilon_{2}}\right\|_{H_{p_{14}}^{\rho}} & \leq\left\|V_{\gamma} *\langle i \nabla\rangle^{\rho}\left(\overline{w^{\varepsilon_{1}}} w^{\varepsilon_{2}}\right)\right\|_{L^{p_{14}}} \leq\left\|\langle i \nabla\rangle^{\rho}\left(\overline{w^{\varepsilon_{1}}} w^{\varepsilon_{2}}\right)\right\|_{L^{p_{15}}} \\
& \leq\left\|w^{\varepsilon_{1}}\right\|_{H_{p_{11}}^{\rho}}\left\|w^{\varepsilon_{2}}\right\|_{L^{p_{12}}}+\left\|w^{\varepsilon_{2}}\right\|_{H_{p_{11}}^{\rho}}\left\|w^{\varepsilon_{1}}\right\|_{L^{p_{12}}}
\end{aligned}
$$

since $1+\frac{1}{p_{14}}=\frac{\gamma}{n}+\frac{1}{p_{15}}$ and $\frac{1}{p_{15}}=\frac{1}{p_{11}}+\frac{1}{p_{12}}$. Similarly we have

$$
\left\|V_{\gamma} * \overline{w^{\varepsilon_{1}}} w^{\varepsilon_{2}}\right\|_{L^{p_{24}}} \leq\left\|w^{\varepsilon_{1}}\right\|_{L^{p_{21}}}\left\|w^{\varepsilon_{2}}\right\|_{L^{p_{22}}} .
$$

\section{Proof of Theorem 1.1}

For $1<\gamma<\min \left\{\frac{2(n+1)}{n+2}, \frac{3 n-2}{n+2}\right\}$, we choose

$$
\frac{(n+2)(\gamma+1)}{4 n}+\frac{1}{2}<\beta<\frac{(n+2)(\gamma+1)}{2 n}, q=\left(\frac{2 \beta}{n+2}+\frac{1}{2}-\frac{\gamma+1}{n}\right)^{-1},
$$

They satisfy

$$
1 \leq \beta \leq 2,2<q<\frac{2 n}{n+2(1-\gamma)}, 1<\gamma<\frac{3 n \beta}{n+2} .
$$

Let $\mu=\frac{1}{2}\left(1+\frac{n}{2}\right)\left(1-\frac{2}{q}\right)$, we also have

$$
\mu+\beta-2 \leq 0, \mu \leq \beta-1, \text { and } \quad 0<\mu \leq \frac{1}{2} .
$$

Let $r, p$ and $s$ be chosen as

$$
\frac{2}{r}=\frac{n}{2}\left(1-\frac{2}{q}\right), \frac{2}{p}+\frac{\gamma}{n}=2-\frac{2}{q}, \frac{2}{s}=1-\frac{2}{r} .
$$


The proof of Theorem 1.1(1). Introduce the function space

$$
X=\left\{v=\left(v^{+}, v^{-}\right) \in C\left(R ;\left(L^{2}\left(R^{n}\right)\right)^{2}\right) ; \quad\|v\|_{X}<+\infty\right\}
$$

with the norm

$$
\begin{aligned}
\|v\|_{X}= & \|v\|_{L_{t}^{\infty}\left(R, H^{\beta}\right)}+\|v\|_{L_{t}^{r}\left(R, H_{q}^{\beta-\mu}\right)}+\left\|\partial_{t} v\right\|_{L_{t}^{\infty}\left(R, H^{\beta-1}\right)}+\left\|\partial_{t} v\right\|_{L_{t}^{r}\left(R, L^{q}\right)} \\
& +\|P v\|_{L_{t}^{\infty}\left(R, H^{\beta-1}\right)}+\|P v\|_{L_{t}^{r}\left(R, L^{q}\right)}+\|J v\|_{L_{t}^{\infty}\left(R, H^{\beta-1}\right)} .
\end{aligned}
$$

Denote by $X_{\rho}$ a ball of a radius $\rho>0$ with a center in the origin in the space $X$. Let us consider the linearized version of (2.1)

$$
\left\{\begin{array}{l}
L_{\varepsilon} w^{\varepsilon}=\langle i \nabla\rangle^{-1} F_{\gamma}(v) \\
\left.w^{\varepsilon}\right|_{t=0}=w_{0}^{\varepsilon}
\end{array}\right.
$$

with a given vecter $v=\left(v^{+}, v^{-}\right) \in X_{\rho}$, where

$$
F_{\gamma}(v)=\sum_{\varepsilon_{1}, \varepsilon_{2}, \varepsilon_{3} \in\{+,-\}} C_{\varepsilon_{1} \varepsilon_{2} \varepsilon_{3}}\left(V_{\gamma} * \overline{v^{\varepsilon_{1}}} v^{\varepsilon_{2}}\right) v^{\varepsilon_{3}}
$$

with some given constants $C_{\varepsilon_{1} \varepsilon_{2} \varepsilon_{3}}$. The integration of the linearized Cauchy problem (3.1) with respect to time yields

$$
w^{\varepsilon}=U_{\varepsilon}(t) w_{0}^{\varepsilon}+\sum_{\varepsilon_{1}, \varepsilon_{2}, \varepsilon_{3} \in\{+,-\}} C_{\varepsilon_{1} \varepsilon_{2} \varepsilon_{3}} \Psi_{\varepsilon}\left(\left(V_{\gamma} * \overline{v^{\varepsilon_{1}}} v^{\varepsilon_{2}}\right) v^{\varepsilon_{3}}\right) .
$$

Taking the $L_{t}^{\infty}\left(R ; H^{\beta}\right)$-norm of (3.2), applying the Hölder inequality, Lemma 2.1 and Lemma 2.3, we find

$$
\begin{aligned}
\left\|w^{\varepsilon}\right\|_{L_{t}^{\infty}\left(R ; H^{\beta}\right)} & \leq\left\|U_{\varepsilon}(t) w_{0}^{\varepsilon}\right\|_{L_{t}^{\infty}\left(R ; H^{\beta}\right)}+C \sum_{\varepsilon_{1}, \varepsilon_{2}, \varepsilon_{3} \in\{+,-\}}\left\|\Psi_{\varepsilon}\left(\left(V_{\gamma} * \overline{v^{\varepsilon_{1}}} v^{\varepsilon_{2}}\right) v^{\varepsilon_{3}}\right)\right\|_{L_{t}^{\infty}\left(R ; H^{\beta}\right)} \\
& \leq\left\|w_{0}^{\varepsilon}\right\|_{H^{\beta}}+C \sum_{\varepsilon_{1}, \varepsilon_{2}, \varepsilon_{3} \in\{+,-\}}\left\|\left(V_{\gamma} * \overline{v^{\varepsilon}} v^{\varepsilon_{2}}\right) v^{\varepsilon_{3}}\right\|_{L_{t}^{r^{\prime}}\left(R ; H_{q^{\prime}}^{\beta+\mu-1}\right)}\|\| w_{0}\left\|_{H^{\beta}}+C \sum_{\varepsilon_{1}, \varepsilon_{2}, \varepsilon_{3} \in\{+,-\}}\right\| v^{\varepsilon_{1}}\left\|_{H_{q}^{\beta+\mu-1}}\right\| v^{\varepsilon_{2}}\left\|_{L^{p}}\right\| v^{\varepsilon_{3}}\left\|_{L^{p}}\right\|_{L_{t}^{r^{\prime}}(R)} \\
& \leq\left\|w_{0}\right\|_{H^{\beta}}+C\|v\|_{L_{t}^{r}\left(R ; H_{q}^{\beta-\mu}\right)}\|v\|_{L_{t}^{s}\left(R ; L^{p}\right)}^{2} \\
& \leq\left\|w_{0}\right\|_{H^{\beta}}+C \rho\|v\|_{L_{t}^{s}\left(R ; L^{p}\right)}^{2}
\end{aligned}
$$

since $p>2>q^{\prime}, q>2>q^{\prime}, \mu \leq \frac{1}{2}$ and $2-\frac{2}{q}=\frac{\gamma}{n}+\frac{2}{p}$. Similarly, taking the $L_{t}^{r}\left(R ; H_{q}^{\beta-\mu}\right)$ we obtain

$$
\begin{aligned}
\left\|w^{\varepsilon}\right\|_{L_{t}^{r}\left(R ; H_{q}^{\beta-\mu}\right)} & \leq\left\|U_{\varepsilon}(t) w_{0}^{\varepsilon}\right\|_{L_{t}^{r}\left(R ; H_{q}^{\beta-\mu}\right)}+C \sum_{\varepsilon_{1}, \varepsilon_{2}, \varepsilon_{3} \in\{+,-\}}\left\|\Psi_{\varepsilon}\left(\left(V_{\gamma} * \overline{v^{\varepsilon_{1}}} v^{\varepsilon_{2}}\right) v^{\varepsilon_{3}}\right)\right\|_{L_{t}^{r}\left(R ; H_{q}^{\beta-\mu}\right)} \\
& \leq\left\|w_{0}^{\varepsilon}\right\|_{H^{\beta}}+C \sum_{\varepsilon_{1}, \varepsilon_{2}, \varepsilon_{3} \in\{+,-\}}\left\|\left(V_{\gamma} * \overline{v^{\varepsilon_{1}}} v^{\varepsilon_{2}}\right) v^{\varepsilon_{3}}\right\|_{L_{t}^{r^{\prime}}\left(R ; H_{q^{\prime}}^{\beta+\mu-1}\right)} \\
& \leq\left\|w_{0}^{\varepsilon}\right\|_{H^{\beta}}+C\|v\|_{L_{t}^{r}\left(R ; H_{q}^{\beta+\mu-1}\right)}\|v\|_{L_{t}^{s}\left(R ; L^{p}\right)}^{2} \\
& \leq\left\|w_{0}\right\|_{H^{\beta}}+C \rho\|v\|_{L_{t}^{s}\left(R ; L^{p}\right)}^{2}
\end{aligned}
$$


since $\mu \leq \frac{1}{2}, p>2>q^{\prime}, q>2>q^{\prime}$ and $2-\frac{2}{q}=\frac{\gamma}{n}+\frac{2}{p}$. Applying the operator $\partial_{t}$ to (3.1) we deduce that $\partial_{t} w^{\varepsilon}$ satisfies the following system

$$
\left\{\begin{array}{l}
L_{\varepsilon} \partial_{t} w^{\varepsilon}=\langle i \nabla\rangle^{-1} \partial_{t} F_{\gamma}(v) \\
\left.\partial_{t} w^{\varepsilon}\right|_{t=0}=-i \varepsilon\langle i \nabla\rangle w_{0}^{\varepsilon}-\left.i\langle i \nabla\rangle^{-1} F_{\gamma}(v)\right|_{t=0}
\end{array}\right.
$$

with

$$
F_{\gamma}(v)=\sum_{\varepsilon_{1}, \varepsilon_{2}, \varepsilon_{3} \in\{+,-\}} C_{\varepsilon_{1} \varepsilon_{2} \varepsilon_{3}}\left(V_{\gamma} * \overline{v^{\varepsilon_{1}}} v^{\varepsilon_{2}}\right) v^{\varepsilon_{3}}
$$

Then by integrating with respect to time,

$$
\partial_{t} w^{\varepsilon}=U_{\varepsilon}(t)\left(\left.\partial_{t} w^{\varepsilon}\right|_{t=0}\right)+\Psi_{\varepsilon}\left(\partial_{t} F_{\gamma}(v)\right) .
$$

Taking the $L_{t}^{\infty}\left(R ; H^{\beta-1}\right)$-norm and $L_{t}^{r}\left(R, L^{q}\right)$-norm, applying the Hölder inequality and Lemma 2.1 we find that, since $\beta \geq 1, \mu \leq \beta-1$ and $\mu+\beta-2 \leq 0$,

$$
\begin{aligned}
& \left\|\partial_{t} w^{\varepsilon}\right\|_{L_{t}^{\infty}\left(R ; H^{\beta-1}\right)}+\left\|\partial_{t} w^{\varepsilon}\right\|_{L_{t}^{r}\left(R ; L^{q}\right)} \\
\leq & \left\|\left.\partial_{t} w^{\varepsilon}\right|_{t=0}\right\|_{H^{\beta-1}}+\left\|\partial_{t} F_{\gamma}(v)\right\|_{L_{t}^{r^{\prime}}\left(R, H_{q^{\prime}}^{\mu+\beta-2}\right)} \\
\leq & \left\|\left.\partial_{t} w^{\varepsilon}\right|_{t=0}\right\|_{H^{\beta-1}} \\
& \quad+C \sum_{\varepsilon_{1}, \varepsilon_{2}, \varepsilon_{3} \in\{+,-\}}\left\|\left(V_{\gamma} *\left(\overline{\partial_{t} v^{\varepsilon_{1}}} v^{\varepsilon_{2}}+\overline{v^{\varepsilon_{1}}} \partial_{t} v^{\varepsilon_{2}}\right)\right) v^{\varepsilon_{3}}+\left(V_{\gamma} * \overline{v^{\varepsilon}} v^{\varepsilon_{2}}\right) \partial_{t} v^{\varepsilon_{3}}\right\|_{L_{t}^{r^{\prime}}\left(R ; L^{q^{\prime}}\right)} \\
& \leq\left\|\left.\partial_{t} w^{\varepsilon}\right|_{t=0}\right\|_{H^{\beta-1}}+C\left\|\partial_{t} v\right\|_{L_{t}^{r}\left(R, L^{q}\right)}\|v\|_{L_{t}^{s}\left(R, L^{p}\right)}^{2} \\
\leq & \left\|\left.\partial_{t} w^{\varepsilon}\right|_{t=0}\right\|_{H^{\beta-1}}+C \rho\|v\|_{L_{t}^{s}\left(R, L^{p}\right)}^{2}
\end{aligned}
$$

On the other hand, we have

$$
\left\|\left.\partial_{t} w^{\varepsilon}\right|_{t=0}\right\|_{H^{\beta-1}} \leq\left\|w_{0}^{\varepsilon}\right\|_{H^{\beta}}+\left\|F_{\gamma}(v)\right\|_{L_{t}^{\infty}\left(R, H^{\beta-2}\right)},
$$

and for $p_{1}>2$ satisfying $\frac{3}{2}=\frac{\gamma}{n}+\frac{3}{p_{1}}$,

$$
\begin{aligned}
\left\|F_{\gamma}(v)\right\|_{L_{t}^{\infty}\left(R, H^{\beta-2}\right)} & \left.\leq C \sum_{\varepsilon_{1}, \varepsilon_{2}, \varepsilon_{3} \in\{+,-\}} \|\left(V_{\gamma} * \overline{v^{\varepsilon_{1}}} v^{\varepsilon_{2}}\right) v^{\varepsilon_{3}}\right) \|_{L_{t}^{\infty}\left(R ; L^{2}\right)} \\
& \leq C\|v\|_{L_{t}^{\infty}\left(R ; L^{p_{1}}\right)}^{3} \leq C\|v\|_{L_{t}^{\infty}\left(R ; H^{\beta}\right)}^{3} \leq C \rho^{3}
\end{aligned}
$$

since $\beta \leq 2, \gamma \leq 3 \beta$ and $\|v\|_{L^{p_{1}}} \leq C\|v\|_{H^{\beta}}$. Then

$$
\left\|\partial_{t} w^{\varepsilon}\right\|_{L_{t}^{\infty}\left(R ; H^{\beta-1}\right)}+\left\|\partial_{t} w^{\varepsilon}\right\|_{L_{t}^{r}\left(R ; L^{q}\right)} \leq C\left\|w_{0}\right\|_{H^{\beta}}+C \rho^{3}+C \rho\|v\|_{L_{t}^{s}\left(R, L^{p}\right)}^{2} .
$$

Notice that $P=t \nabla+x \partial_{t}, J_{\varepsilon}=\langle i \nabla\rangle x+i \varepsilon t \nabla$ and $L_{\varepsilon}=i \partial_{t}-\varepsilon\langle i \nabla\rangle$. We get

$$
\begin{gathered}
J_{\varepsilon}=i \varepsilon P-\varepsilon L_{\varepsilon},\left[L_{\varepsilon}, P\right]=-i \varepsilon\langle i \nabla\rangle^{-1} \nabla L_{\varepsilon}, \\
{[x,\langle i \nabla\rangle]=\langle i \nabla\rangle^{-1} \nabla,\left[P,\langle i \nabla\rangle^{-1}\right]=\langle i \nabla\rangle^{-3} \nabla \partial_{t}}
\end{gathered}
$$

and

$$
P\left(\left(V_{\gamma} * \overline{v^{\varepsilon_{1}}} v^{\varepsilon_{2}}\right) v^{\varepsilon_{3}}\right)=\left(V_{\gamma} * \overline{v^{\varepsilon_{1}}} v^{\varepsilon_{2}}\right) P\left(v^{\varepsilon_{3}}\right)+\left(t \nabla V_{\gamma} * \overline{v^{\varepsilon_{1}}} v^{\varepsilon_{2}}\right) v^{\varepsilon_{3}} .
$$


Applying the operator $P$ to (3.1) yields

$$
\left\{\begin{array}{l}
L_{\varepsilon} P w^{\varepsilon}=i \varepsilon\langle i \nabla\rangle^{-2} \nabla F_{\gamma}(v)-\langle i \nabla\rangle^{-1} P F_{\gamma}(v)-\langle i \nabla\rangle^{-3} \nabla \partial_{t} F_{\gamma}(v) \\
\left.P w^{\varepsilon}\right|_{t=0}=\left.x \partial_{t} w^{\varepsilon}\right|_{t=0}=x\left(-i \varepsilon\langle i \nabla\rangle w_{0}^{\varepsilon}-\left.i\langle i \nabla\rangle^{-1} F_{\gamma}(v)\right|_{t=0}\right)
\end{array}\right.
$$

with

$$
P F_{\gamma}(v)=\sum_{\varepsilon_{1}, \varepsilon_{2}, \varepsilon_{3} \in\{+,-\}} C_{\varepsilon_{1} \varepsilon_{2} \varepsilon_{3}}\left(V_{\gamma} * \overline{v^{\varepsilon_{1}}} v^{\varepsilon_{2}}\right) P v^{\varepsilon_{3}}+\left(t \nabla V_{\gamma} * \overline{v^{\varepsilon_{1}}} v^{\varepsilon_{2}}\right) v^{\varepsilon_{3}}
$$

Integrating with respect to time, we get

$$
P w^{\varepsilon}=U_{\varepsilon}(t)\left(\left.P w^{\varepsilon}\right|_{t=0}\right)-\Psi_{\varepsilon}\left(i \varepsilon\langle i \nabla\rangle^{-1} \nabla F_{\gamma}(v)\right)+\Psi_{\varepsilon}\left(P F_{\gamma}(v)\right)+\Psi_{\varepsilon}\left(\langle i \nabla\rangle^{-2} \nabla \partial_{t} F_{\gamma}(v)\right) .
$$

Taking the $L_{t}^{\infty}\left(R ; H^{\beta-1}\right)$-norm and the $L_{t}^{r}\left(R, L^{q}\right)$-norm of (3.6), applying the Hölder inequality and Lemma 2.1, we find

$$
\begin{gathered}
\left\|P w^{\varepsilon}\right\|_{L_{t}^{\infty}\left(R ; H^{\beta-1}\right)}+\left\|P w^{\varepsilon}\right\|_{L_{t}^{r}\left(R, L^{q}\right)} \\
\leq\left\|\left.P w^{\varepsilon}\right|_{t=0}\right\|_{H^{\beta-1}}+\left\|\langle i \nabla\rangle^{-1} \nabla F_{\gamma}\right\|_{L_{t}^{r^{\prime}}\left(R, L^{q^{\prime}}\right)} \\
\quad+\left\|P F_{\gamma}(v)\right\|_{L_{t}^{r^{\prime}}\left(R ; L^{q^{\prime}}\right)}+\left\|\langle i \nabla\rangle^{-2} \nabla \partial_{t} F_{\gamma}(v)\right\|_{L_{t}^{r^{\prime}}\left(R ; L^{q^{\prime}}\right)} \\
\leq\left\|\left.P w^{\varepsilon}\right|_{t=0}\right\|_{H^{\beta-1}}+\left\|F_{\gamma}(v)\right\|_{L_{t}^{r^{\prime}}\left(R, L q^{\prime}\right)} \\
\quad+\left\|P F_{\gamma}(v)\right\|_{L_{t}^{r^{\prime}\left(R ; L^{q^{\prime}}\right)}}+\left\|\partial_{t} F_{\gamma}(v)\right\|_{L_{t}^{r^{\prime}}\left(R ; L^{q^{\prime}}\right)}
\end{gathered}
$$

since $\beta \geq 1$ and $\mu+\beta-2 \leq 0$ and $\mu \leq \beta-1$. As in the proof of (3.5) we deduce

$$
\begin{aligned}
& \left\|F_{\gamma}(v)\right\|_{L_{t}^{r^{\prime}}\left(R, L^{q^{\prime}}\right)}+\left\|\partial_{t} F_{\gamma}(v)\right\|_{L_{t}^{r^{\prime}}\left(R ; L^{q^{\prime}}\right)} \\
\leq & C\|v\|_{L_{t}^{r}\left(R, L^{q}\right)}\|v\|_{L_{t}^{s}\left(R, L^{p}\right)}^{2}+C\left\|\partial_{t} v\right\|_{L_{t}^{r}\left(R, L^{q}\right)}\|v\|_{L_{t}^{s}\left(R, L^{p}\right)}^{2} \leq C \rho\|v\|_{L_{t}^{s}\left(R, L^{p}\right)}^{2} .
\end{aligned}
$$

Let $p_{3}>2$ and $s_{3}>2$ satisfy

$$
\frac{3}{2}-\frac{1}{q}=\frac{\gamma+1}{n}+\frac{2}{p_{3}}, 1-\frac{1}{r}=\frac{2}{s_{3}} .
$$

The Hölder inequality and Lemma 2.3 imply

$$
\begin{aligned}
& \left\|P F_{\gamma}(v)\right\|_{L_{t}^{r^{\prime}}\left(R ; L^{q^{\prime}}\right)} \\
\leq & \left.\left.C \sum_{\varepsilon_{1}, \varepsilon_{2}, \varepsilon_{3} \in\{+,-\}}\left[\|\left(V_{\gamma} * \overline{v^{\varepsilon}} v^{\varepsilon_{2}}\right) P v^{\varepsilon_{3}}\right)\left\|_{L_{t}^{r^{\prime}}\left(R ; L^{q^{\prime}}\right)}+\right\|\left(t \nabla V_{\gamma} * \overline{v^{\varepsilon_{1}}} v^{\varepsilon_{2}}\right) v^{\varepsilon_{3}}\right) \|_{L_{t}^{r^{\prime}\left(R ; L^{q^{\prime}}\right)}}\right] \\
\leq & C\|P v\|_{L_{t}^{r}\left(R, L^{q}\right)}\|v\|_{L_{t}^{s}\left(R, L^{p}\right)}^{2}+C\|v\|_{L_{t}^{\infty}\left(R, L^{2}\right)}\left\|t^{1 / 2} v\right\|_{L_{t}^{s_{3}}\left(R, L^{p_{3}}\right)}^{2} \\
\leq & C \rho\|v\|_{L_{t}^{s}\left(R, L^{p}\right)}^{2}+C \rho\left\|t^{1 / 2} v\right\|_{L_{t}^{s_{3}}\left(R, L^{p_{3}}\right)}^{2}
\end{aligned}
$$

here we use the condition $\left\|\nabla V_{\gamma}\right\| \leq C|x|^{-(\gamma+1)}$. By Lemma 2.2 we have

$$
\begin{aligned}
\|v\|_{L_{t}^{s}\left(R, L^{p}\right)} & \leq C\left\|\langle t\rangle^{-\frac{n}{2}\left(1-\frac{1}{p}\right)}\left(\|v\|_{H^{\alpha}}+\|J v\|_{H^{\alpha-1}}\right)\right\|_{L_{t}^{s}(R)} \\
& \leq C\left(\|v\|_{L_{t}^{\infty}\left(R, H^{\beta}\right)}+\|J v\|_{L_{t}^{\infty}\left(R, H^{\beta-1}\right)}\right) \leq C \rho
\end{aligned}
$$


since $\alpha=\left(1+\frac{n}{2}\right)\left(1-\frac{2}{p}\right) \leq \beta$ and $\frac{n}{2}\left(1-\frac{2}{p}\right)>\frac{1}{s}$. Similarly,

$$
\begin{aligned}
\left\|t^{1 / 2} v\right\|_{L_{t}^{s_{3}}\left(R, L^{p_{3}}\right)} & \leq C\left\|\langle t\rangle^{-\frac{n}{2}\left(1-\frac{1}{p_{3}}\right)+\frac{1}{2}}\left(\|v\|_{H^{\alpha_{3}}}+\|J v\|_{H^{\alpha_{3}-1}}\right)\right\|_{L_{t}^{s_{3}}(R)} \\
& \leq C\left(\|v\|_{L_{t}^{\infty}\left(R, H^{\beta}\right)}+\|J v\|_{L_{t}^{\infty}\left(R, H^{\beta-1}\right)}\right) \leq C \rho,
\end{aligned}
$$

since $\alpha_{3}=\left(1+\frac{n}{2}\right)\left(1-\frac{2}{p_{3}}\right) \leq \beta$ and $\frac{n}{2}\left(1-\frac{2}{p_{3}}\right)>\frac{1}{s_{3}}$. Then we obtain, from (3.7)-(3.11),

$$
\begin{aligned}
& \left\|P w^{\varepsilon}\right\|_{L_{t}^{\infty}\left(R ; H^{\beta-1}\right)}+\left\|P w^{\varepsilon}\right\|_{L_{t}^{r}\left(R, L^{q}\right)} \leq\left\|\left.P w^{\varepsilon}\right|_{t=0}\right\|_{H^{\beta-1}}+C \rho^{3}, \\
& \left\|w^{\varepsilon}\right\|_{L_{t}^{\infty}\left(R ; H^{\beta}\right)}+\left\|w^{\varepsilon}\right\|_{L_{t}^{r}\left(R ; H_{q}^{\beta-\mu}\right)} \leq\left\|w_{0}^{\varepsilon}\right\|_{H^{\beta}}+C \rho^{3}, \\
& \left\|\partial_{t} w^{\varepsilon}\right\|_{L_{t}^{\infty}\left(R ; H^{\beta-1}\right)}+\left\|\partial_{t} w^{\varepsilon}\right\|_{L_{t}^{r}\left(R ; L^{q}\right)} \leq\left\|w_{0}^{\varepsilon}\right\|_{H^{\beta}}+C \rho^{3},
\end{aligned}
$$

To estimate the term $\left\|\left.P w^{\varepsilon}\right|_{t=0}\right\|_{H^{\beta-1}}$, we give some estimates. It follows from the Sobolev embedding theorem that

$$
\begin{aligned}
\left\|F_{\gamma}(v)\right\|_{L_{t}^{\infty}\left(R, L^{2}\right)} & \leq C \sum_{\varepsilon_{1}, \varepsilon_{2}, \varepsilon_{3} \in\{+,-\}}\left\|\left(V_{\gamma} * \overline{v^{\varepsilon_{1}}} v^{\varepsilon_{2}}\right) v^{\varepsilon_{3}}\right\|_{L_{t}^{\infty}\left(R, L^{2}\right)} \\
& \leq\|v\|_{L_{t}^{\infty}\left(R, L^{p_{5}}\right)}^{3} \leq C\|v\|_{L_{t}^{\infty}\left(R, H^{\beta}\right)}^{3} \leq C \rho^{3},
\end{aligned}
$$

where $p_{5}=\frac{6 n}{3 n-2 \gamma}$, which satisfies $p_{5} \leq \frac{2 n}{n-2 \beta}$ because of $\gamma \leq 3 \beta$. Using the relation $x=\langle i \nabla\rangle^{-1} J_{\varepsilon}-i \varepsilon t\langle i \nabla\rangle^{-1} \nabla$ we deduce

$$
\begin{aligned}
& \left\|x F_{\gamma}(v)\right\|_{L_{t}^{\infty}\left(R, L^{2}\right)} \leq C \sum_{\varepsilon_{1}, \varepsilon_{2}, \varepsilon_{3} \in\{+,-\}}\left\|\left(V_{\gamma} * \overline{v^{\varepsilon_{1}}} v^{\varepsilon_{2}}\right)\left(x v^{\varepsilon_{3}}\right)\right\|_{L_{t}^{\infty}\left(R, L^{2}\right)} \\
\leq & \left.\left.C \sum_{\varepsilon_{1}, \varepsilon_{2}, \varepsilon_{3} \in\{+,-\}}\|\| v^{\varepsilon_{1}}\left\|_{L^{p_{4}}}\right\|\left\|v^{\varepsilon_{2}}\right\|_{L^{p_{4}}}\left(\|\langle i \nabla\rangle^{-1} J_{\varepsilon} v^{\varepsilon_{3}}\right)\left\|_{L^{p_{4}}}+t\right\|\langle i \nabla\rangle^{-1} \nabla v^{\varepsilon_{3}}\right) \|_{L^{p_{4}}}\right) \|_{L_{t}^{\infty}(R)} \\
\leq & C\|v\|_{L_{t}^{\infty}\left(R, L^{\left.p_{4}\right)}\right.}^{2}\left\|\langle i \nabla\rangle^{-1} J v\right\|_{L_{t}^{\infty}\left(R, L^{p_{4}}\right)}+C\left\|t^{1 / 3} v\right\|_{L_{t}^{\infty}\left(R, L^{p_{4}}\right)}^{3} \\
\leq & C\|v\|_{L_{t}^{\infty}\left(R, H^{\beta}\right)}^{2}\|J v\|_{L_{t}^{\infty}\left(R, H^{\beta-1}\right)}+C\left(\|v\|_{L_{t}^{\infty}\left(R, H^{\beta}\right)}+\|J v\|_{L_{t}^{\infty}\left(R, H^{\beta-1}\right)}\right)^{3} \\
\leq & C\left(\rho+\|J v\|_{L_{t}^{\infty}\left(R, H^{\beta-1}\right)}\right)^{3} \leq C \rho^{3},
\end{aligned}
$$

where $p_{4}=\frac{6 n}{3 n-2 \gamma}$, which satisfies

$$
2<p_{4} \leq \frac{2 n}{n-2 \beta}, \frac{n}{2}\left(1-\frac{2}{p_{4}}\right) \geq \frac{1}{3},\left(1+\frac{n}{2}\right)\left(1-\frac{2}{p_{4}}\right) \leq \beta
$$

because of $1<\gamma \leq \frac{3 n \beta}{n+2}$. Using the relation $\left[\langle i \nabla\rangle^{\beta-1}, x\right]=-(\beta-1)\langle i \nabla\rangle^{\beta-3} \nabla$ we deduce

$$
\begin{aligned}
& \left\|\left.P w^{\varepsilon}\right|_{t=0}\right\|_{H^{\beta-1}} \leq\left\|x\langle i \nabla\rangle w_{0}^{\varepsilon}\right\|_{H^{\beta-1}}+\left\|x\langle i \nabla\rangle^{-1} F_{\gamma}(v)\right\|_{L_{t}^{\infty}\left(R, H^{\beta-1}\right)} \\
\leq & \left\|x\langle i \nabla\rangle w_{0}^{\varepsilon}\right\|_{H^{\beta-1}}+\left\|\langle i \nabla\rangle^{-1} x F_{\gamma}(v)\right\|_{L_{t}^{\infty}\left(R, H^{\beta-1}\right)}+\left\|\langle i \nabla\rangle^{-3} \nabla F_{\gamma}(v)\right\|_{L_{t}^{\infty}\left(R, H^{\beta-1}\right)} \\
\leq & \left\|\langle i \nabla\rangle^{\beta-1} x\langle i \nabla\rangle w_{0}^{\varepsilon}\right\|_{L^{2}}+C\left\|x F_{\gamma}(v)\right\|_{L_{t}^{\infty}\left(R, L^{2}\right)}+C\left\|F_{\gamma}(v)\right\|_{L_{t}^{\infty}\left(R, L^{2}\right)} \\
\leq & \left\|\langle x\rangle\langle i \nabla\rangle^{\beta} w_{0}^{\varepsilon}\right\|_{L^{2}}+C \rho^{3}+C\left(\rho+\|J v\|_{L_{t}^{\infty}\left(R, H^{\beta-1}\right)}\right)^{3} \\
\leq & \left\|w_{0}\right\|_{H^{\beta, 1}}+C \rho^{3},
\end{aligned}
$$


which, combining with (3.12), yields

$$
\left\|P w^{\varepsilon}\right\|_{L_{t}^{\infty}\left(R ; H^{\beta-1}\right)}+\left\|P w^{\varepsilon}\right\|_{L_{t}^{r}\left(R, L^{q}\right)} \leq\left\|w_{0}\right\|_{H^{\beta, 1}}+C \rho^{3} .
$$

Notice that

$$
\left[L_{\varepsilon}, x\right]=-\varepsilon\langle i \nabla\rangle^{-1} \nabla,\left[x,\langle i \nabla\rangle^{-1}\right]=-\langle i \nabla\rangle^{-3} \nabla .
$$

Then we deduce that $x w^{\varepsilon}$ satisfies

$$
L_{\varepsilon}\left(x w^{\varepsilon}\right)=-\varepsilon\langle i \nabla\rangle^{-1} \nabla w^{\varepsilon}-\langle i \nabla\rangle^{-1}\left(x F_{\gamma}(v)\right)+\langle i \nabla\rangle^{-1} \nabla F_{\gamma}(v) .
$$

Using $J_{\varepsilon}=i \varepsilon P-\varepsilon L_{\varepsilon} x$ and (3.13) yields

$$
\left\|J_{\varepsilon} w^{\varepsilon}\right\|_{L_{t}^{\infty}\left(R, H^{\beta-1}\right)} \leq\left\|P w^{\varepsilon}\right\|_{L_{t}^{\infty}\left(R, H^{\beta-1}\right)}+\left\|L_{\varepsilon}\left(x w^{\varepsilon}\right)\right\|_{L_{t}^{\infty}\left(R, H^{\beta-1}\right)},
$$

with

$$
\begin{aligned}
& \left\|L_{\varepsilon}\left(x w^{\varepsilon}\right)\right\|_{L_{t}^{\infty}\left(R, H^{\beta-1}\right)} \\
\leq & \left\|w^{\varepsilon}\right\|_{L_{t}^{\infty}\left(R, H^{\beta-1}\right)}+\left\|\langle i \nabla\rangle^{-2} F_{\gamma}(v)\right\|_{L_{t}^{\infty}\left(R, H^{\beta-1}\right)}+\left\|\langle i \nabla\rangle^{-1}\left(x F_{\gamma}(v)\right)\right\|_{L_{t}^{\infty}\left(R, H^{\beta-1}\right)} \\
\leq & \left\|w^{\varepsilon}\right\|_{L_{t}^{\infty}\left(R, H^{\beta}\right)}+\left\|F_{\gamma}(v)\right\|_{L_{t}^{\infty}\left(R, L^{2}\right)}+\left\|x F_{\gamma}(v)\right\|_{L_{t}^{\infty}\left(R, L^{2}\right)} \leq C\left\|w_{0}\right\|_{H^{\beta}}+C \rho^{3} .
\end{aligned}
$$

Then we get

$$
\left\|J_{\varepsilon} w^{\varepsilon}\right\|_{L_{t}^{\infty}\left(R, H^{\beta-1}\right)} \leq C\left\|w_{0}\right\|_{H^{\beta, 1}}+C \rho^{3} .
$$

A combination of (3.12) with (3.13), (3.14), (3.17) and (3.18) yields

$$
\|w\|_{X} \leq C\left\|w_{0}\right\|_{H^{\beta, 1}}+C \rho^{3} .
$$

Therefore the map $M: w=M(v)$ defined by the problem (3.1), transforms a ball $X_{\rho}$ with a small radius $\rho=C\left\|w^{0}\right\|_{H^{\beta, 1}}$ into itself. Denote $\tilde{w}=M(\tilde{v})$, then in the same way as in the proof of (3.19) we have

$$
\|M(v)-M(\tilde{v})\|_{X} \leq C \rho^{2}\|v-\tilde{v}\|_{X}
$$

Thus $M$ is a contraction mapping in $X_{\rho}$ and so there exists a unique solution $w=M(w)$ of (3.1) if the norm $\left\|w^{0}\right\|_{H^{\beta, 1}}$ is small enough.

To prove the asymptotic of the solution $w(t, x)$, we use the equation, for $|t|>\left|t^{\prime}\right|$,

$$
U_{\varepsilon}(-t) w^{\varepsilon}(t)-U_{\varepsilon}\left(-t^{\prime}\right) w^{\varepsilon}\left(t^{\prime}\right)=\int_{t^{\prime}}^{t} U_{\varepsilon}(-\tau)\langle i \nabla\rangle^{-1} F_{\gamma}(w(\tau)) d \tau
$$

Taking the $H^{\beta}$-norm of this equation, using the similar proof of (3.3) and (3.4), we deduce

$$
\left\|U_{\varepsilon}(-t) w^{\varepsilon}(t)-U_{\varepsilon}\left(-t^{\prime}\right) w^{\varepsilon}\left(t^{\prime}\right)\right\|_{H^{\beta}} \leq C \rho^{2}\left\langle t^{\prime}\right\rangle^{-\delta}
$$

with $\delta=\frac{2 n \beta}{n+2}-2>0$, since we have $\|w\|_{X} \leq \rho$ and

$$
\left\|\langle t\rangle^{-\frac{n}{2}\left(1-\frac{1}{p}\right)}\right\|_{L_{t}^{s}\left(\left[t^{\prime}, t\right]\right)}^{2} \leq C\left\langle t^{\prime}\right\rangle^{-\delta}
$$

Then there uniquely exist finial states $w_{ \pm}^{\varepsilon} \in H^{\beta}$ satisfying, for $\pm t$ large enough,

$$
\left\|w^{\varepsilon}(t)-U_{\varepsilon}(t) w_{ \pm}^{\varepsilon}\right\|_{H^{\beta}} \leq C \rho^{2}\langle t\rangle^{-\delta}
$$


Set $u(t)=\frac{1}{2}\left(w^{+}(t)-w^{-}(t)\right), f_{ \pm}(x)=\frac{1}{2}\left(w_{ \pm}^{+}-w_{ \pm}^{-}\right), g_{ \pm}(x)=-\frac{i}{2}\langle i \nabla\rangle\left(w_{ \pm}^{+}+w_{ \pm}^{-}\right)$and $u_{ \pm}(t)=\frac{1}{2}\left(U_{+}(t) w_{ \pm}^{+}-U_{-}(t) w_{ \pm}^{-}\right)$. Then $u(t)$ and $u_{ \pm}(t)$ satisfy Theorem 1.1](1).

Proof of Theorem 1.1(2). For given $\left(f_{-}, g_{-}\right) \in X^{\beta, 1}$ and $v=\left\{v^{+}, v^{-}\right) \in X_{\rho}$, we consider the linearized version of the final state problem of (3.1)

$$
\left\{\begin{array}{l}
L_{\varepsilon} w^{\varepsilon}=-\langle i \nabla\rangle^{-1} F_{\gamma}(v) \\
\left\|U_{\varepsilon}(t) w^{\varepsilon}-w_{-}^{\varepsilon}(x)\right\|_{H^{\beta}} \rightarrow 0 \text { as } t \rightarrow \infty
\end{array}\right.
$$

with $w_{-}^{\varepsilon}(x)=i\langle i \nabla\rangle^{-1} g_{-}(x)-\varepsilon f_{-}(x) \in H^{\beta, 1}$. The integration with respective to time yields

$$
w^{\varepsilon}(t)=U_{\varepsilon}(t) w_{-}^{\varepsilon}+\int_{-\infty}^{t} U_{\varepsilon}(t-\tau)\langle i \nabla\rangle^{-1} F_{\gamma}(v(\tau)) d \tau
$$

In the same way as in the proof of Theorem 1.1(1), we find that, if $\left\|\left(f_{-}, g_{-}\right)\right\|_{X^{\beta, 1}} \leq \rho$ small, there uniquely exists a global solution $w^{\varepsilon}(t) \in C\left(R, H^{\beta}\right)$ and a final state $w_{+}^{\varepsilon} \in H^{\beta}$ such that, as $t \rightarrow+\infty$,

$$
\left\|w^{\varepsilon}(t)-U_{\varepsilon}(t) w_{+}^{\varepsilon}\right\|_{H^{\beta}} \leq C\langle t\rangle^{-\delta}
$$

with $\delta=\frac{2 n \beta}{n+2}-2>0$. Set $u(t)=\frac{1}{2}\left(w^{+}(t)-w^{-}(t)\right), f_{+}(x)=\frac{1}{2}\left(w_{+}^{+}-w_{+}^{-}\right), g_{+}(x)=$ $-\frac{i}{2}\langle i \nabla\rangle\left(w_{+}^{+}+w_{+}^{-}\right)$and $u_{+}(t)=\frac{1}{2}\left(U_{+}(t) w_{+}^{+}-U_{-}(t) w_{+}^{-}\right)$. Then $u(t)$ and $u_{+}(t)$ satisfy Theorem 1.1(2).

\section{References}

[1] G. Menzala and W. Strauss, On a wave equation with a cubic convolution, J. Differential Equations, 43 (1982), 93-105.

[2] K. Mochizuki, On small data scattering with cubic convolution nonlinearity, J. Math. Soc. Japan, 41 (1989), 143-160.

[3] K. Hidano, Small data scattering and blow-up for a wave equation with a cubic convolution, Funkcialaj Ekvacioj, 43 (2000), 559-588.

[4] K. Hidano, Small data scattering for the Klein-Gordon equation with a cubic convolution nonlinearity, Discrete Cont. Dyn. Sys., 15(2006), 973-981.

[5] N. Hayashi, P. I. Naumkin, Scattering operator for nonlinear Klein-Gordon equations in higher space dimensions, J. Differential Equations 244(2008), 188-199.

[6] G. Ponce, On the global well-posedness of the Benjamin-Ono equation, Differential and Integral Equations, 4 (1991), 527-542.

[7] S. Klainerman, Global existence of small amplitude solutions to nonlinear KleinGordon equations in four space-time dimensions, Comm. Pure Appl. Math. 38 (1985), 631-641.

[8] B. Marshall, W. Strauss, S. Wainger, $L^{p}-L^{q}$ estimates for the Klein-Gordon equation, J. Math. Pures Appl. 59(1980), 417-440. 